Le secret médical vise à protéger la relation de confiance entre le médecin et son patient - y compris en prison. Et c'est précisément cette confiance, avec les exceptions déjà possibles aujourd'hui, qui permet la meilleure protection de la sécurité publique. C'est pourquoi la FMH soutient la position de l'Association des médecins du canton de Genève, qui vise à protéger le secret médical. Dans ce numéro du BMS, le Dr Pierre-Alain Schneider et le Pr Philippe Ducor, médecin et professeur de droit, éclairent les points de vue médical et juridique, parce que le débat politique a trop sous-estimé l'importance du secret médical et que le rôle du médecin pénitentiaire est souvent mal compris.

Dr Jürg Schlup, président de la FMH

\title{
Expliquer le secret médical
}

\section{Pierre-Alain Schneider}

Président de la Société Médicale de la Suisse Romande (SMSR)

Il y a bien longtemps, je m'étais insurgé en entendant un professeur de médecine parler du serment d'Hippocrate comme d'une tradition périmée. Il voulait sans doute dire que le patient était devenu très transparent face aux assureurs et aux statisticiens. Je lui avais rappelé que ce serment était toujours bien vivant sous la forme de la Déclaration de Genève de l'Association médicale mondiale.

Nos patients nous font confiance parce qu'ils savent qu'ils peuvent compter sur notre discrétion. La volonté de contrôler économiquement notre activité n'y change rien. Le secret est un privilège que nous, professionnels de la santé, partageons avec les avocats et les ecclésiastiques. Son importance et sa valeur sont reconnues depuis des siècles. Il est protégé par le Code pénal, et sa violation n'est pas un délit mineur puisqu'elle peut être sanctionnée par une peine privative de liberté.

Il y a cependant des exceptions qu'il faut connaître, dont l'état de nécessité licite. On peut se réjouir des modifications récentes de plusieurs lois d'application cantonales du Code pénal dans la mesure où elles le rappellent au

\section{Nos patients nous font confiance parce qu'ils savent qu'ils peuvent compter sur le secret médical.}

médecin qui estime devoir parler. Mais lui imposer de rapporter tout fait dont on pourrait a posteriori estimer qu'il était un signe de danger, c'est le transformer en auxiliaire de l'autorité d'exécution et tirer un trait sur le serment d'Hippocrate. La naïveté du législateur fait sourire. Non, le médecin ne lit pas dans les âmes! Et il ne peut remplacer l'application rigoureuse des mesures de détention.

Car ce que le public réclame, c'est la sécurité. Les meurtres de Lucie, Marie et Adeline ont eu un impact émotionnel considérable et personne ne veut voir la répétition de tels drames. Les autorités doivent agir, mais encore doivent-elles le faire avec clairvoyance. Dans l'affaire genevoise, les rapports Ziegler et Chappuis sont clairs: le secret médical n'a joué aucun rôle. On a en revanche fait trop facilement confiance à un détenu qui s'était bien comporté, sans attendre l'expertise psychiatrique qui aurait dû être réalisée. On a oublié la complexité de ces psychopathes. La victime elle-même était une thérapeute et je ne peux croire qu'elle se soit jetée dans la

\section{Imposer au médecin de rapporter, c'est le trans-} former en auxiliaire de l'autorité d'exécution.

gueule du loup si elle avait perçu les signes d'un danger imminent.

Les psychiatres capables d'expliquer certains mécanismes qui poussent ces personnes perturbées au crime, avouent eux-mêmes leurs doutes lorsqu'il s'agit de prévoir leur évolution à long terme. Or notre ordre juridique ne peut se satisfaire d'incertitudes et c'est sa faiblesse en face de tels criminels. Ainsi, la mesure d'internement à vie récemment prononcée contre le meurtrier de Marie pourrait être cassée par l'instance de recours.

Les médecins genevois ont lancé une initiative cantonale pour éliminer l'obligation faite au médecin d'informer et la remplacer par la faculté d'informer du système actuel, sans risque de sanction lorsqu'il s'agit de détenus dangereux. L'enjeu sera d'expliquer en quoi le secret médical contribue à la sécurité, et pourquoi il faut laisser au médecin la liberté de décider quand il est opportun de signaler un fait inquiétant. La campagne sera difficile et devra aussi convaincre que l'obligation d'informer entretient l'illusion dangereuse que la récidive peut être prévenue. N’oublions pas qu'avec ces détenus exceptionnels, on joue toujours avec le feu! 